\title{
STUDY OF TEMPLATE COMPRESSION IN IMAGE CORRELATION BASED MOTION ESTIMATION
}

\author{
Hema Tekwani \\ Research Scholar, Electronics Engineering Department, \\ Harcourt Butler Technical University, Kanpur, India \\ Dr. Krishna Raj \\ Professor, Electronics Engineering Department, \\ Harcourt Butler Technical University, Kanpur, India
}

\begin{abstract}
The aim of present study was to develop a template tracking based image correlation algorithm for small-scale motion estimation. Image correlation based motion estimation algorithms consume significant data due to storage of high-resolution image sequence. We propose a template tracking based image correlation algorithm coupled with wavelet based data compression technique. We configured a two-camera system stereovision setup for synchronized image acquisition. The setup calibration produced camera parameters to implement in the proposed correlation algorithm. We obtained hand motion sequences from the setup and correlated using the proposed algorithm to obtain template tracking parameter (TTP) as a measure of correlation. To investigate the effect of data compression on correlation, we correlated the compressed motion frames using compressed templates with bior 4.4, bior 5.5 and bior 6.8 compression levels. Inclusion of data compression in the proposed algorithm produced TTP value close to those obtained with uncompressed templates. TTP value using bior 6.8 was 0.9493 and 0.8608 for left and right camera, which was close to uncompressed image frames. The correlation results were visualized using Paraview visualization tool to validate the proposed algorithm.
\end{abstract}

Key words: Compression, Image acquisition, Image correlation, Motion estimation, Non-contact, Template tracking

Cite this Article: Hema Tekwani and Krishna Raj, Study of Template Compression in Image Correlation Based Motion Estimation, International Journal of Electrical Engineering and Technology (IJEET), 12(6), 2021, pp. 308-319.

https://iaeme.com/Home/issue/IJEET? Volume $=12 \&$ Issue $=6$ 


\section{INTRODUCTION}

Pattern recognition in computer vision and image processing is an excellent method in motion estimation (ME) and has gained attention in past decade. These techniques are more pronounced for slow motion when the motion information content does not change significantly in subsequent frames. Optical flow based estimation have gained an attention in last decade [1]. Block matching techniques include motion vectors, by the selection and identification of blocks in moving frames. Inclusion of robust estimators and intense computations wiener based pelrecursive estimator were introduced with minimization of computation time, but were not effective for large displacements. Computational complexity increases with large displacements for multi-resolution motion measurement. Shin et al. [2] proposed the feature based tracking algorithm. The motion is analyzed with object features since the search space is not always informative. Moreover, the installation of any motion sensor on the object is also inapt on most cases. Few studies reported with data and space minimization in ME. Image registration that includes information of motion in moving frames plays an important role in optical flow based motion identification and quantification [3]. High speed image processing was proposed in few studies since the installation of physical sensor is inapt sometimes. These techniques are essential where small-scale displacements are concerned that includes pixel or sub-pixel information. However, such techniques involve complicated set-ups and require peer calibrations [4]. Bradley et al. [5] produced the accurate 3D face image, but the limitation lies in the complicated set-up.

Non-contact ME technique works on tracking the local information in the motion frames. 3D motion capture was studied using motion stereo with multiple cameras [6]. Guo et al. [7] proposed the algorithm for the measurement of displacement of large-scale structures. The computational efficiency increases in stereovision configuration for $3 \mathrm{D}$ measurements [8]. Image correlation based non-contact method involves multiple steps. It includes formation of identical templates, with the help of the image captured from reference camera. The templates are correlated with captured images to predict both in-plane and out of plane object motion. Multiple cameras are preferred for out of plane motion capture followed by template based feature tracking. Multiple templates tracking (TT) carry the motion information content. The size of the templates also plays an important role in tracking the pixel level displacement. In addition to TT, the efficiency of image based ME techniques also depend on lighting and contrast. The lighting contrast should be uniform during calibration and image acquisition. Cameras should be synchronized [9] to overcome acquisition delay and to reduce correlation errors. While the image correlation based ME algorithms are rigorously studied, they consume significant data space. This is due to the storage of high-resolution image sequences. Prediction of micro displacements with pixel and sub-pixel accuracy consumes lot of data. It is essential to employ data compression techniques prior to correlation algorithm without affecting the information content in moving frames. Wavelets have been emerged as a powerful tool in the field of image and video compression. Wavelets employ a multi-level image-processing algorithm for ME. With multiple level of decomposition, wavelet allows image compression with minimum number of pixels required without losing the image details [10]. The biorthogonal wavelet has linear phase, which is an essential feature for image reconstruction. In addition, the multi-resolution analysis using bi-orthogonal wavelet allows the decomposition of image at various levels [11]. The aim of current investigation was to develop an effective image correlation algorithm coupled with wavelet based data compression technique based on TT based image correlation. The algorithm traces the location of multiple templates in moving hand frames. The templates are produced from a reference image (preferably the first from captured images). Three distinct levels of bi-orthogonal wavelets bior 4.4, bior 5.5 and bior 6.8 are considered for compression of motion frames. A template tracking parameter (TTP) is 
obtained from the correlation of uncompressed and compressed image sequences. The TTP obtained from bior 6.8 compression was close to that obtained from uncompressed image correlation. We propose that the data compression technique can be coupled with image correlation algorithm for efficient ME. The correlation results obtained from coupled algorithm are validated from the motion visualization with both uncompressed and compressed frames.

\section{CORRELATION BASED MOTION ESTIMATION COUPLED WITH DATA COMPRESSION}

\subsection{Correlation Based Motion Estimation}

Camera captured image sequences are correlated using predefined templates since the algorithm works on the principle of correlating identical templates in motion frames. Frame-wise information content in ME is significantly small or approximately constant when image correlation based techniques are involved. Single camera is sufficient for in-plane measurement while two or more cameras are preferred for out of plane motion. Prior to correlation, the ME set-up is calibrated to obtain intrinsic and extrinsic parameters [12]. During calibration and correlation, the occluded region can also considered for flow estimation [13] with the use of two or more cameras. The calibration grid is set in focus to avoid the stereographic errors [14]. To predict the motion in correlation algorithm a series of co-ordinate transformations from world to sensor co-ordinate system are involved. The displacement in sensor coordinates $\left(x_{p}, y_{p}\right)$ is given in equation (1) and (2), where $\left(X_{W}, Y_{W}, Z_{W}\right)$ is the world co-ordinates, $\left(c_{x}, c_{y}\right)$ is the center of image plane, $\left(f_{x}, f_{y}\right)$ is the coordinates of focal length and $[R]$ and $[T]$ is the rotation and translation vector [9, 12]. Figure 1 shows the principle of algorithm for single camera, where $w$ signifies the world coordinate system with the camera coordinate system $(X, Y, Z)$. Several coordinate transformations are involved. First transformation is from world coordinate system to camera coordinate system, second from camera coordinate system to image coordinate system and the third from image coordinate system to sensor coordinate system.

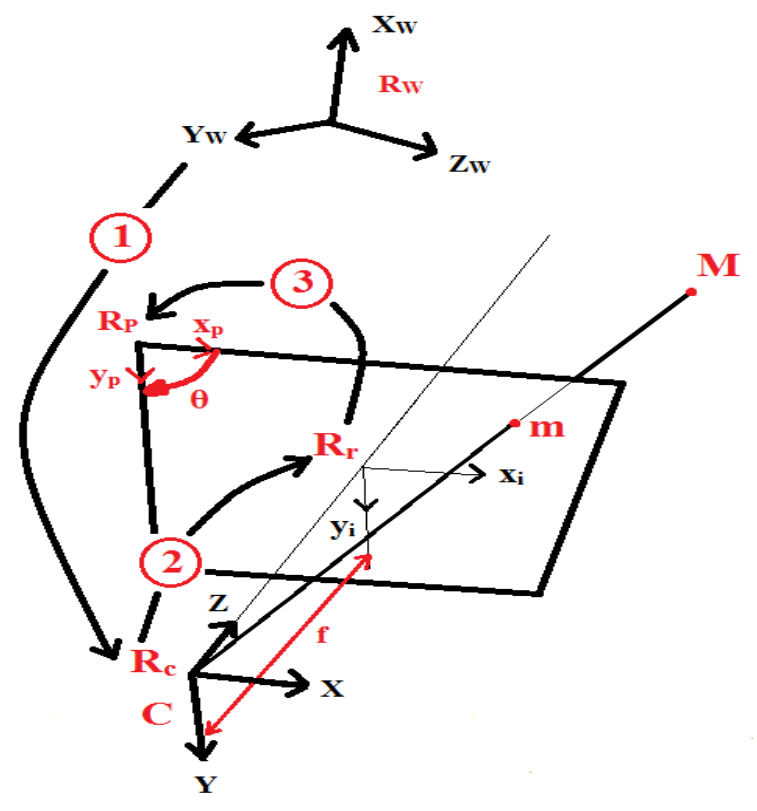

Figure 1 Principle of Image Correlation 


$$
\begin{gathered}
x_{p}=\frac{c_{x}\left(R_{31} X_{W}+R_{32} Y_{W}+t_{z}\right)+f_{x}\left(R_{11} X_{W}+R_{12} Y_{W}+t_{x}\right)}{R_{31} X_{W}+R_{32} Y_{W}+t_{z}} \\
y_{p}=\frac{c_{y}\left(R_{31} X_{W}+R_{32} Y_{W}+t_{z}\right)+f_{y}\left(R_{21} X_{W}+R_{22} Y_{W}+t_{y}\right)}{R_{31} X_{W}+R_{32} Y_{W}+t_{z}}
\end{gathered}
$$

\subsubsection{Template Tracking Parameter}

The suitability of correlation algorithm depends on how efficiently it correlates the templates in moving frames. Identical templates are formed from a reference image that should preferably be the first of captured images. We considered a template size of 10 pixels during the entire ME algorithm both with and without the data compression scheme. Efficiency of pixel tracking in ME increases by introducing more number of templates provided the average template intensity should not be below a threshold value. To avoid this, we selected threshold intensity of 10 during the algorithm with lower intensity variation. We correlated 70 templates in the process of correlation. The TTP is a dimensionless quantity and comes out unity for a perfect match. For $j$ observations of variables $x$ and $y$, the template tracking in terms of correlation can be expressed as in Equation (3), where $x^{\prime}$ and $y^{\prime}$ are the mean values.

$$
\operatorname{Cor}(x, y)=\frac{\sum_{j=1}^{m}\left(x_{j}-x^{\prime}\right)\left(y_{j}-y^{\prime}\right)}{\sqrt{\sum_{j=1}^{m}\left(x_{j}-x^{\prime}\right)^{2} \sum_{j=1}^{m}\left(y_{j}-y^{\prime}\right)^{2}}}
$$

\subsection{Wavelet Based Compression}

Wavelet analyses the information content in images using multi-resolution analysis. Multiresolution formulation [15] enables image decomposition with finer details [16]. Symmetric filters are preferred in the image reconstruction to minimize the edge error effects. Discrete wavelet transform is an effective tool for image compression resulting in LL, LH, HL, and HH sub-bands. The bi-orthogonal wavelet family is preferred for the compression as compared to the orthogonal ones. The symmetric output given by the bi-orthogonal filters, when presented with symmetric input is advantageous in image compression. The compression algorithms in bi-orthogonal wavelets uses hierarchical based wavelet scheme known as set partitioning in hierarchical trees algorithm. The algorithm initiates with the creation of list of insignificant sets, pixels and significant pixels [17]. The pixel values in the image have very high degree of correlation among rows and columns. Linear phase is important in many image-processing applications for compression to avoid unexpected distortions in the compressed image. Because of such admissibility, the original image reconstruction is effective after decomposition. With lossless compression the original image should ideally retain, which cannot be always true after decomposition. Some unperceived errors are admissible in lossy compression. De-noising eliminates errors up to some extent and therefore lossy compression is acceptable [18].

\subsection{Proposed Algorithm: Coupling of Data Compression with Image Correlation}

The cameras are oriented such that the object is visible and maintained in focus. Camera system calibration is a primitive step before motion estimation. The Camera Calibrator App in MATLAB performs stereo calibration of images captured from two camera system and produce intrinsic and extrinsic parameters as shown in Table 1. Figure 2 represents the ME algorithm developed and proposed in present study. We developed template code, auto-cropping code and correlation code using MATLAB environment in this study. The algorithm starts with the generation of identical square templates for both compressed and uncompressed images. A template code develops templates from the first image of reference camera (left camera in our case). The auto-cropping code eliminates the out of interest area from motion frames. An image 
correlation code correlates the templates with motion frames. The whole algorithm is also associated with the data compression scheme. We performed hand motion experiment by giving small displacement and captured the movement with 15 consecutive frames is acquired in moving frames using the camera system. Table 2 represents the experimental details. Next step is the formation of compressed and uncompressed templates. Selection of the template size can vary as per the information needed. We developed separate templates for compressed and uncompressed images. After template making, the correlation program with the calibration parameters generated the TTP data for both uncompressed and compressed frames.

\subsubsection{Compression Effects on Template Tracking}

The main idea behind compression is to de-correlate the pixels [19]. The formation of templates plays a major role in TTP. A threshold value is set prior to the template formation. To obtain the compressed data set, we applied bi-orthogonal wavelet with three distinct compression levels known as bior 4.4, bior 5.5 and bior 6.8. Similar to the uncompressed one, we performed compressed image correlation using compressed templates. TTP values from the compressed image correlation are the measure of effective data compression implemented in the proposed algorithm.

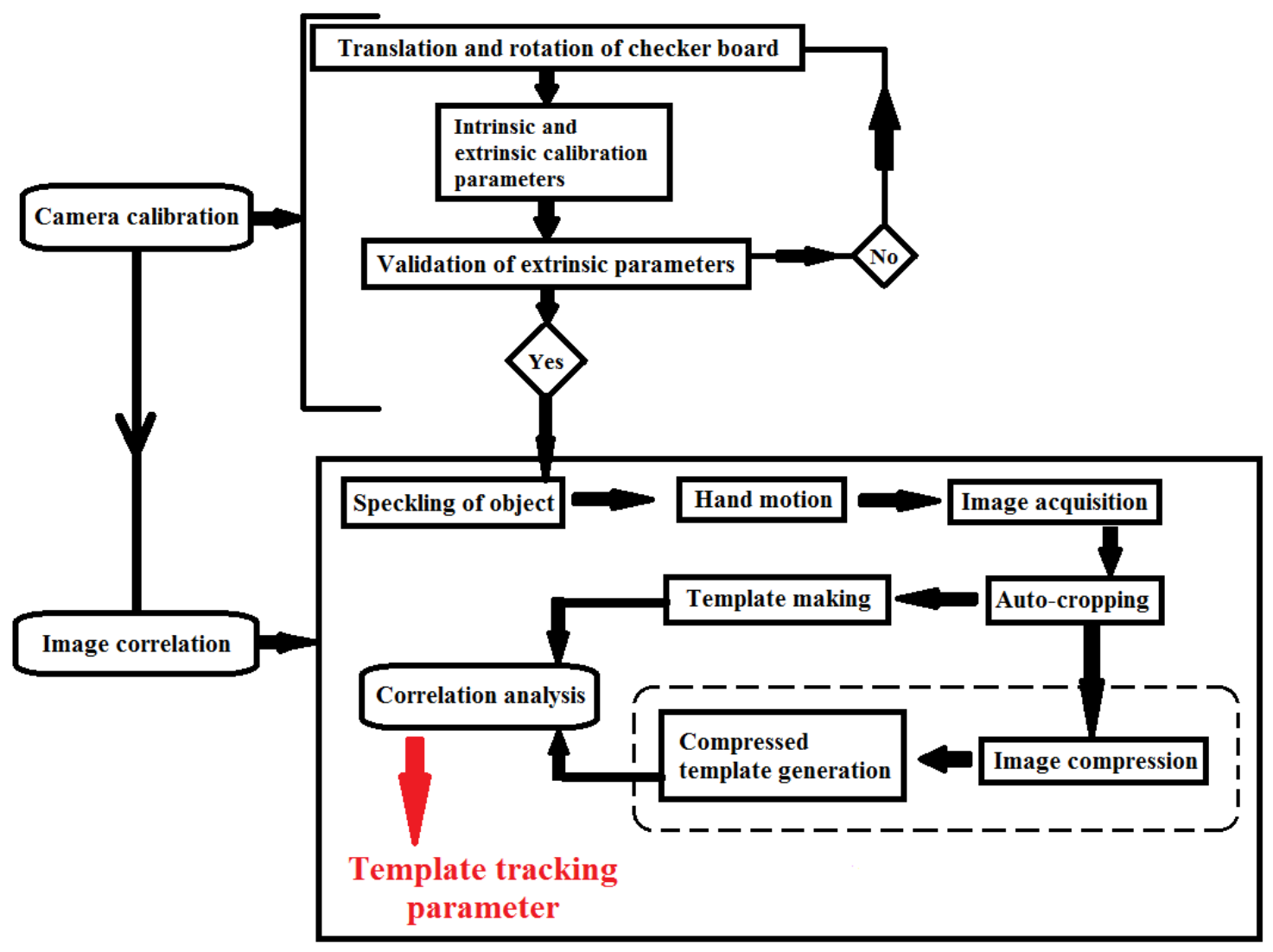

Figure 2 Image Correlation Algorithm Coupled with Data Compression

\section{EXPERIMENTAL}

\subsection{Set-up Calibration}

The experimental set-up includes the set of high-speed cameras (Olympus ispeed TR, 10000fps) with $50 \mathrm{~mm}$ Nikkor lenses, a rigid support structure where the cameras are mounted and inclined towards the object under consideration. The set-up is also equipped with two light sources (1000w) to ensure sufficient lighting during testing. We calibrated high-speed cameras in a 
stereo system as a single measurement panel [12]. The Camera Calibrator App in MATLAB calibrates the checkerboard pattern images acquired from synchronized cameras. We selected left camera as reference during our entire analysis. Small random translation and rotation of the checkerboard plate (9x7grid) is sufficient for calibration. We acquired 30 images with single frame capture setting at $1500 \mathrm{fps}$, which includes translation and rotation both.

The calibration parameters such as focal length and principal point are essential parameters and produced during the calibration process. These are the intrinsic and extrinsic camera parameters and obtained as mentioned in Section 2.1. The calibration process determined the pixel level accuracy of proposed algorithm. The produced calibration parameters are further used to produce the world coordinates using transformation equations [12]. These equations are used to convert 2D sensor coordinates to the 3D coordinates relative to the camera world coordinate system, so the position of the cameras need to be unaltered in the entire calibration.

Table 1 Set-up Calibration Parameters

\begin{tabular}{|c|c|c|}
\hline Name & Parameter & Details \\
\hline \multirow{2}{*}{ Camera-1 } & Principal Point & $(679.533,528.911) \mathrm{Px}$ \\
\cline { 2 - 3 } & Focal Length & $(2811.811,2742.701) \mathrm{Px}$ \\
\hline \multirow{2}{*}{ Camera-2 } & Principal Point & $(661.277,513.228) \mathrm{Px}$ \\
\cline { 2 - 3 } & Focal Length & $(2731.799,2711.544) \mathrm{Px}$ \\
\hline
\end{tabular}

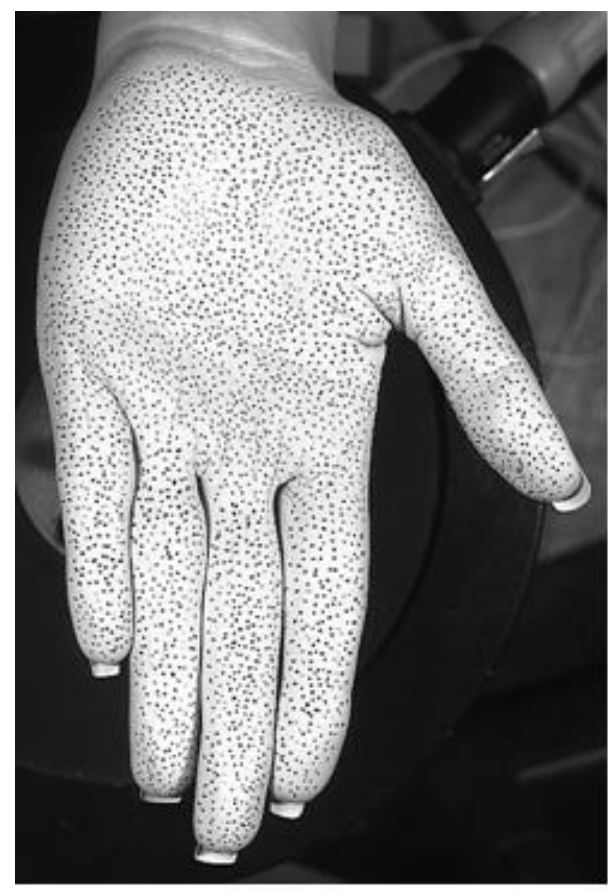

(a)

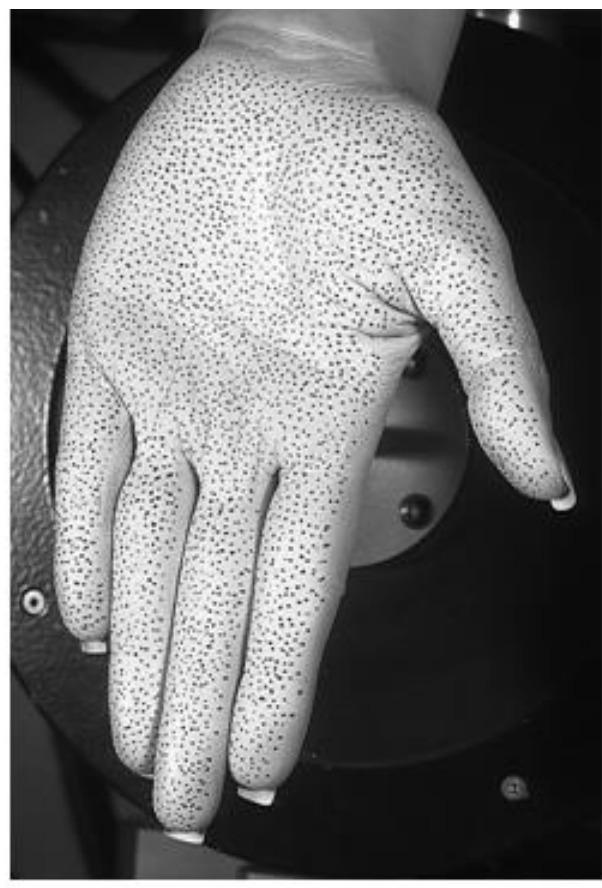

(b)

Figure 3 Uncompressed Image Frames for Hand Motion from (a) Left Camera (b) Right Camera

\subsection{Hand Motion Case}

After the calibration process, we performed the hand motion experiment by giving small displacement. Constant illumination ensures the effectiveness of correlation [20]. We used two 
light sources for each camera. The motion of the human hand is captured simultaneously with the same camera settings adopted during calibration. Table 2 shows the constraints for ME. Figure 3 shows the hand motion frames captured during experiment. The two image frames shows the first frame captured from camera 1 and camera 2. Figure 4 shows the compressed frames of hand motion. Total 15 frames were captured for hand motion at 1500fps. Figure 3 and 4 represents the first captured frame with and without compression respectively. After image acquisition, the next part was to execute the correlation algorithm. The parameters obtained during the calibration procedure are incorporated in the correlation algorithm as mentioned in the next section.

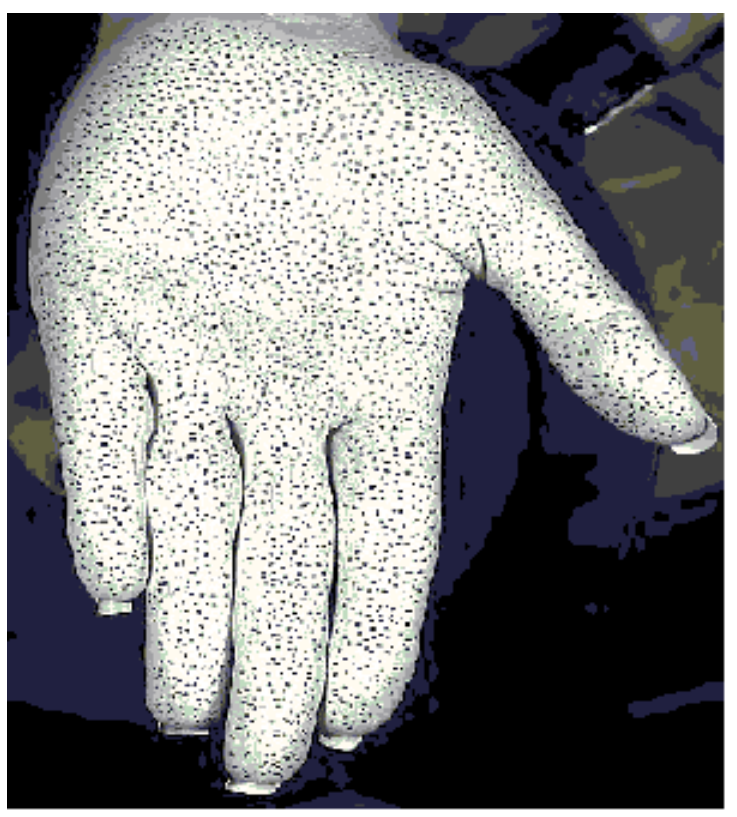

(a)

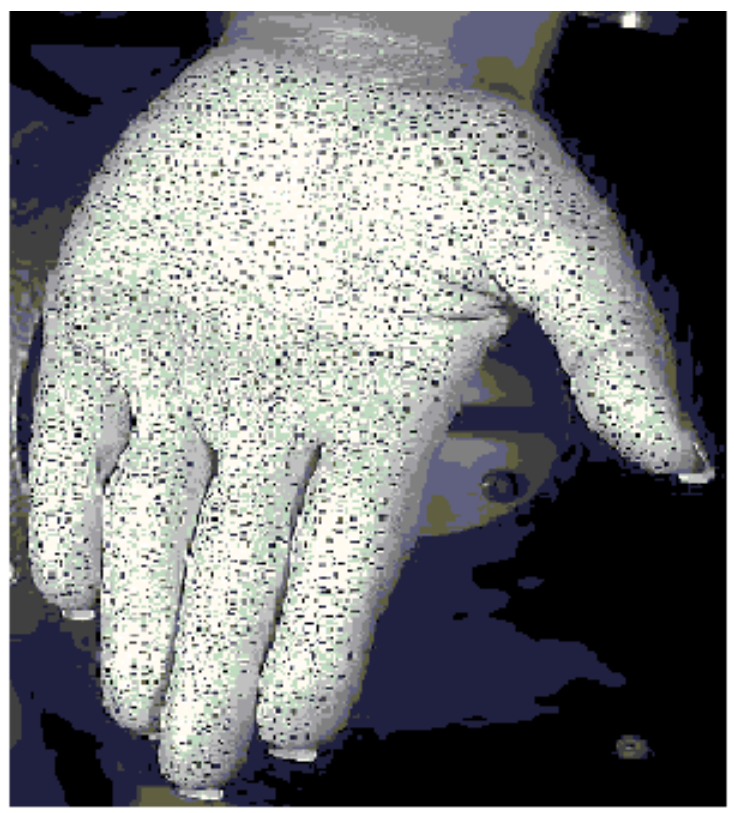

(b)

Figure 4 Compressed Image Frames for Hand Motion from (a) Left Camera (b) Right Camera

Table 2 ME Algorithm Constraints

\begin{tabular}{|c|c|c|}
\hline Name & Parameter & Details \\
\hline \multirow{4}{*}{ Constants } & Shutter speed & $1500 \mathrm{fps}$ \\
\cline { 2 - 3 } & Frames & 15 \\
\cline { 2 - 3 } & Template count & 70 \\
\cline { 2 - 3 } & Template Size & 10 \\
\hline \multirow{2}{*}{$\begin{array}{c}\text { Data Compression } \\
\text { Technique }\end{array}$} & Bior Compression & Bior 6.8 \\
\cline { 2 - 3 } & & Bior 5.5 \\
\cline { 2 - 3 }
\end{tabular}

\subsection{Implementation of Correlation Algorithm}

We developed 70 templates from the reference images for each motion case. These templates are developed separately for the uncompressed and compressed cases. We selected the first frame of camera 1 (left camera) as reference for template making. As mentioned in Table 2, an identical template size of 10 pixels generates 70 templates in total. These templates were called in the correlation algorithm in both the cases i.e. with and without data compression scheme as mentioned in Figure 2. Similar to original uncompressed templates, the compressed templates were also generated from reference compressed image. The frames were compressed with bior levels as mentioned in Table 2. We obtained the TTP after the correlation algorithm and compared it in between the uncompressed and compressed cases. 


\section{RESULTS AND DISCUSSION}

\subsection{Accuracy Analysis of Template Tracking Technique}

Verification of ME setup is essential when the motion is to be predicted with image processing techniques. Since the proposed technique traces the template location in subsequent images, we investigated the pixel level accuracy through rigorous calibration procedure. The calibration error is represented in Figure 5 which indicate overall mean error was found to be 0.18 pixel which indicated 0.82 pixel accuracy in both camera 1 and camera 2 . The pixel size at the sensor plane of the high-speed camera is $21 \mu \mathrm{m}$ as per the camera specifications [21]. Hence, the calibration error of 0.18 pixel corresponds to $3.78 \mu \mathrm{m}$. This indicates the accuracy of our proposed algorithm. The obtained pixel level accuracy is significant for TT algorithm.

\subsection{Effect of Data Compression on Template Tracking}

Table 3 shows the TTP calculated for 15 frames with 70 templates for both uncompressed and compressed images. TTP values are evaluated for both the cameras. It was observed that TTP produced by camera 1 and camera 2 were close to unity which showcase good correlation of templates in motion frames. The values were reported 0.9911 and 0.8923 for camera 1 and camera 2 respectively. Correlation of compressed images with bior 6.8 produced the TTP values as 0.9493 and 0.8608 for camera 1 and camera 2 respectively. We observed that even if the images are compressed, the net reduction in TTP was $4.21 \%$ and $3.53 \%$ compared to uncompressed image for camera 1 and 2 respectively as shown in Figure 6.

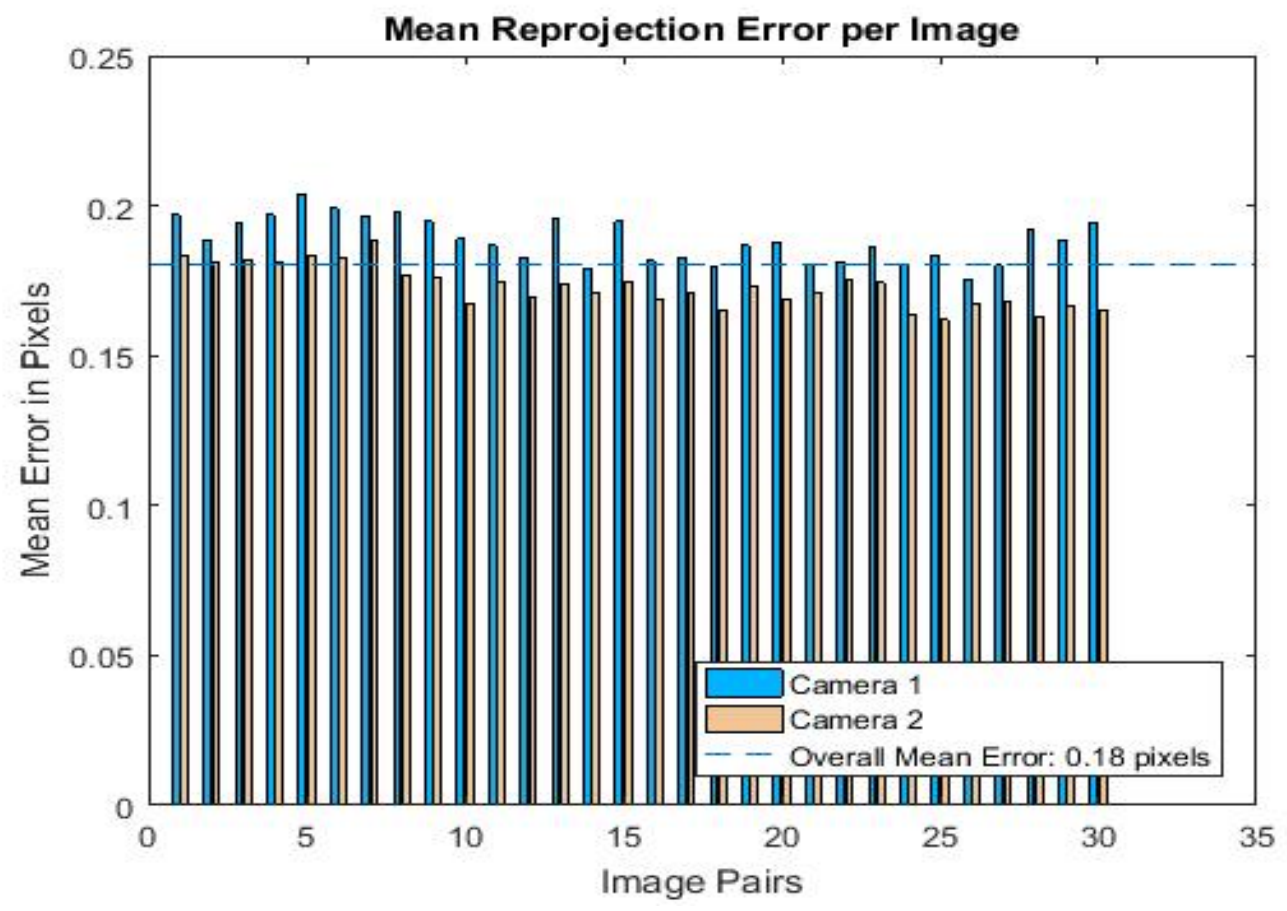

Figure 5 Error Estimation in Calibration Frames

Hence, it can be concluded that with bior 6.8 level data compression, the correlation is well established in between motion sequences. Further investigation on compression effects revealed that with bior 5.5 level compression, the TTP was reduced to 0.8351 and 0.7626 . This indicates $15.74 \%$ and $14.53 \%$ reduction compared to original uncompressed image correlation. Bior 4.4 represented $24.58 \%$ and $22.16 \%$ TTP reduction compared to uncompressed image correlation. Both of these two lower levels of compression were not significant in image correlation. Hence, bior 6.8 is proposed as a correlation effective compression. 
Table 3 Compression Effects on Template Tracking

\begin{tabular}{|c|c|c|c|c|}
\hline Frame Details & $\begin{array}{c}\text { Compression } \\
\text { Level }\end{array}$ & $\begin{array}{c}\text { TT in Frames } \\
\text { (No. of Templates x } \\
\text { No. of Frames) }\end{array}$ & $\begin{array}{c}\text { TTP } \\
\text { (Camera 1) }\end{array}$ & $\begin{array}{c}\text { TTP } \\
\text { (Camera 2) }\end{array}$ \\
\hline Uncompressed & - & $70 \times 15$ & 0.9911 & 0.8923 \\
\hline Compressed & Bior 6.8 & $70 \times 15$ & 0.9493 & 0.8608 \\
\hline Compressed & Bior 5.5 & $70 \times 15$ & 0.8351 & 0.7626 \\
\hline Compressed & Bior 4.4 & $70 \times 15$ & 0.7474 & 0.6945 \\
\hline
\end{tabular}

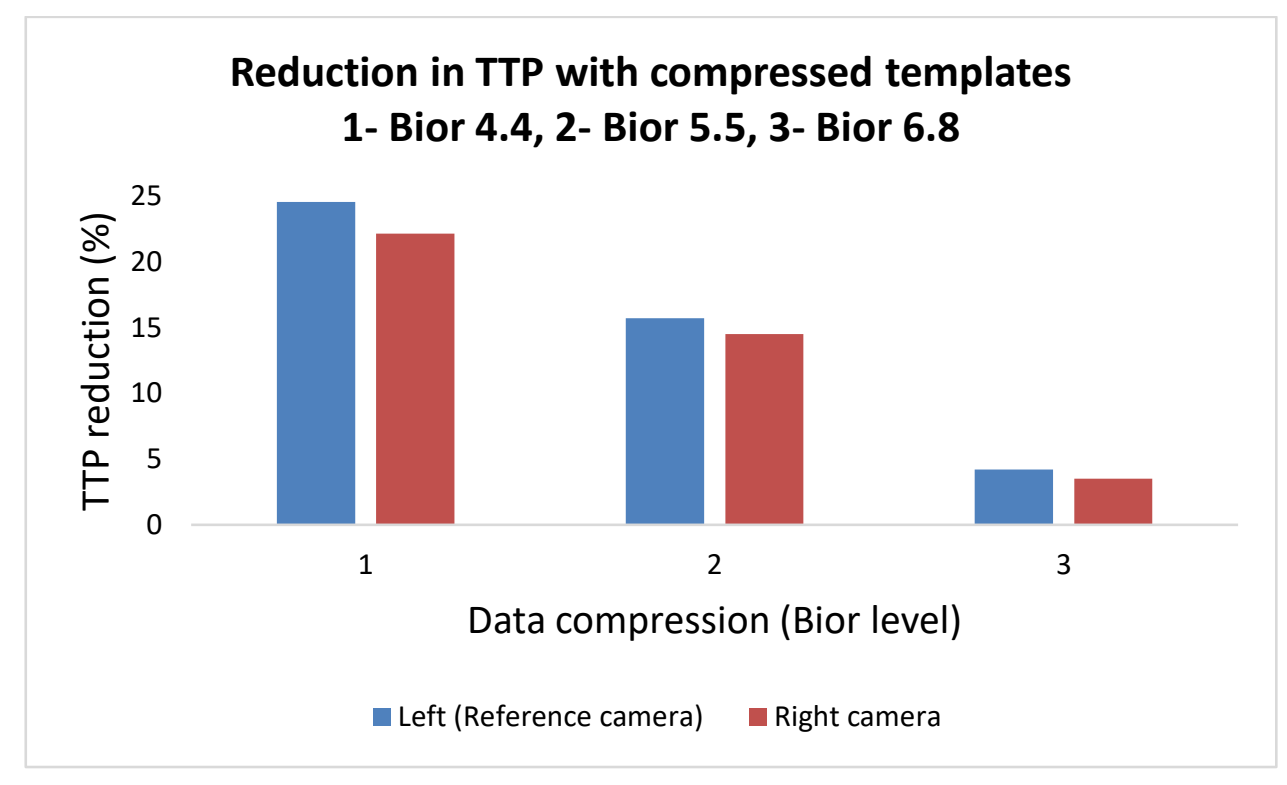

Figure 6 Reduction in TTP with Compressed Templates

\section{MOTION VISUALIZATION AND VALIDATION}

The correlation results from hand motion are visualized in open source data visualization software Paraview 5.6 to validate the motion predicted from compressed and uncompressed data set. We obtained the TTP data and displacement results from correlation algorithm. The results are $\mathrm{X}$ and $\mathrm{Y}$ displacement data from the correlation algorithm and were imported in Paraview in 1:1 scale. Figure 7 and 8 represents the displacement field obtained by TT based algorithm. Figure 7 indicates motion predicted by correlating the uncompressed templates on motion frames whereas Figure 8 showcase the same using bior 6.8 compressed templates. It is evident that the displacement field predicted by bior 6.8 compressed image correlation agree with uncompressed case. The obtained values are close to unity, which also signifies good correlation. Investigation of motion predicted from bior 6.8 compressed templates as depicted in Figure 6 further validates the efficacy of the data compression technique. 


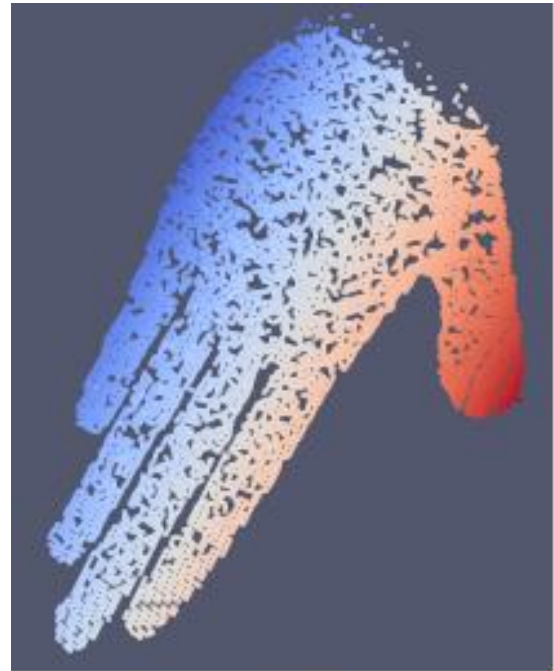

(a)

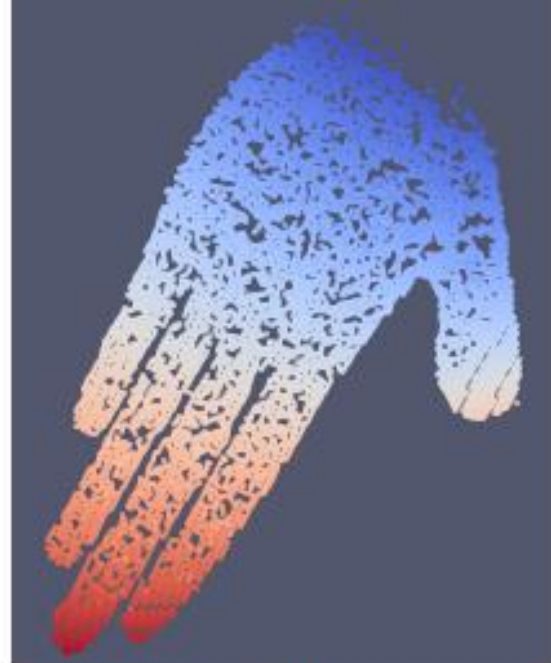

(b)

Figure 7 Motion Visualization for Uncompressed Frames

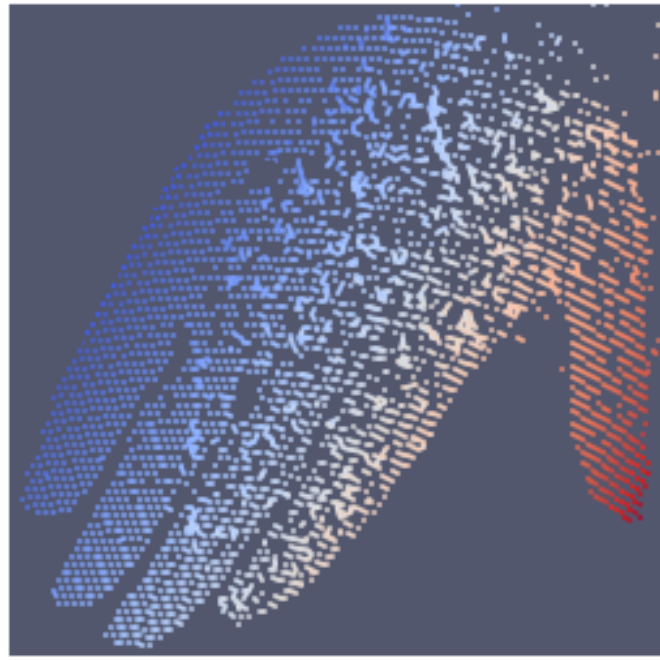

(a)

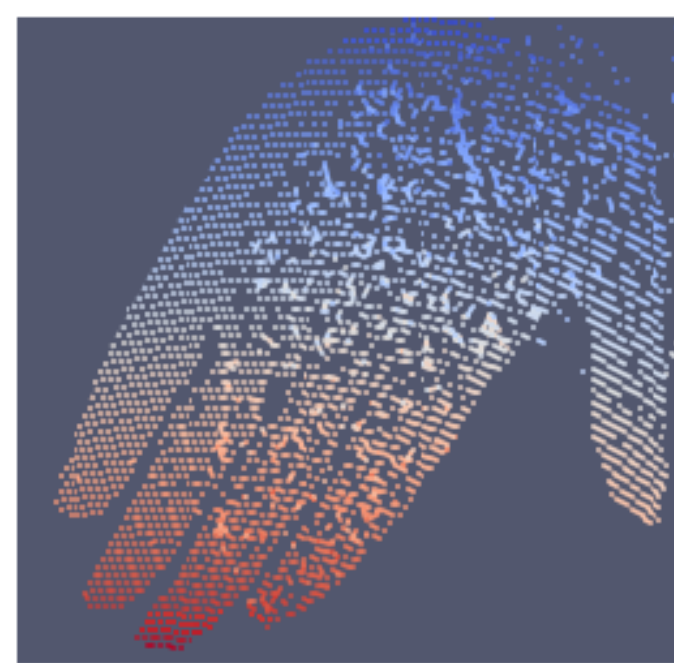

(b)

Figure 8 Motion Visualization for Compressed Frames

\section{CONCLUSION}

Conventional image processing techniques in ME consumes significant space due to the storage of pixel level motion information in moving frames. Correlation based $\mathrm{ME}$ algorithm coupled with data compression is developed and proposed in present investigation. We configured a stereovision camera system for image based motion acquisition. We used wavelet based data compression technique to compress image frames prior to correlation and compared them with the correlation results obtained from uncompressed frames. Wavelets bior 4.4, bior 5.5 and bior 6.8 were considered to produce compressed data for image correlation. We compared compressed and uncompressed data in terms of TTP. The proposed algorithm when used without compression produce the TTP value of 0.9911 and 0.8923 from left and right cameras, which signifies a good correlation of templates in the corresponding frames. Correlation of images with bior 6.8 compressed templates revealed $4.21 \%$ and $3.53 \%$ reduction in TTP, which was the lowest TTP reduction among all the compression levels considered i.e. bior 4.4, bior 5.5 and bior 6.8. Hence, it can be concluded that bior 6.8 compressed templates correlation can 
effectively predict motion equivalent to uncompressed templates using image correlation algorithm.

\section{ACKNOWLEDGEMENT}

The authors sincerely acknowledge the experimental facilities at Structures Lab at IIT Kanpur, and Department of Electronics Engineering at HBTU Kanpur. Financial support for QIP from AICTE, New Delhi is appreciated.

\section{REFERENCES}

[1] D. Marius, and S. Nedevschi, Motion Estimation Using the Correlation Transform, IEEE Trans. Image Process., 22, 2013, 3260-3270.

[2] J. Shin, S. Kim, S. Kang, S.W. Lee, J. Paik, B. Abidi, and M. Abidi, Optical Flow-Based RealTime Object Tracking Using Non-Prior Training Active Feature Model, Real-Time Imaging, 11, 2005, 204-218.

[3] Y. Kellar, and A. Averbuch, Global Parametric Image Alignment via High-Order Approximation, Computer Vision and Image Understanding, 109, 2007, 244-259.

[4] H. Nguyen, H. Kieu, Z. Wang, and H.N.D. Le, Three-Dimensional Facial Digitization Using Advanced Digital Image Correlation, Applied Optics, 57, 2018, 2188-2196.

[5] D. Bradley, W. Heidrich, T. Popa, and A. Sheffer, High Resolution Passive Facial Performance Capture, ACM Transactions on Graphics, 29, 2010, 1-10.

[6] S. Vedula, S. Baker, P. Rander, R. Collins, and T. Kanade, Three-Dimensional Scene Flow, IEEE Trans. Pattern Analysis Machine Intell., 27, 2005, 475-480.

[7] J. Guo, and C. Zhu, Dynamic Displacement Measurement of Large-Scale Structures Based on the Lucas-Kanade Template Tracking Algorithm, Mech. Sys. Signal Process., 66-67, 2016, 425436.

[8] Y. Gao, T. Cheng, Y. Su, X. Xu, Y. Zhang, and Q. Zhang, High-Efficiency and High-Accuracy Digital Image Correlation for Three-Dimensional Measurement, Optics and Lasers in Engineering, 65, 2014, 73-80.

[9] E.M.C. Jones, and M.A. Iadicola, A Good Practices Guide for Digital Image Correlation, International Digital Image Correlation Society, 2018.

[10] M.S. Song, Wavelet Image Compression, Contemporary Mathematics, 2003.

[11] P.M.K. Prasad, and G. Umamadhuri, Biorthogonal Wavelet-Based Image Compression, Artificial Intelligence and Evolutionary Computations in Engineering Systems, Advances in Intelligent Systems and Computing, 668, 2018, Springer.

[12] M.A. Sutton, J.J. Orteu, H.W. Schreier, Image Correlation for Shape, Motion and Deformation Measurements, 2009, Springer.

[13] J. Xiao, H. Cheng, H. Sawhney, C. Rao, and M. Isnardi, Bilateral Filtering-Based Optical Flow Estimation with Occlusion Detection, In European Conference On Computer Vision, 2006, 211 224. 
[14] P. Reu, Calibration: A Good Calibration Image, Society for Experimental Mechanics, 37, 2013, $1-3$.

[15] X.M. Ma, A Revised Edge Detection Algorithm Based on Wavelet Transform for Coal Gangue Image, In IEEE ICMLC conference, 2007, 1639-1642.

[16] P.M.K. Prasad, D.Y.V. Prasad, and Sasibhushana Rao, Performance Analysis of Orthogonal and Biorthogonal Wavelets for Edge Detection of X-ray Images, Procedia Computer Science, 87, 2016, 116-121.

[17] M.C. Stamm, and K.J.R. Liu, Wavelet Based Image Compression Anti-Forensics, In $17^{\text {th }}$ International Conference On Image Processing, Hong-Kong, 2010, 1737-1740.

[18] J.S. Walker, and T.Q. Nguyen, Wavelet-Based Image Compression, CRC Press LLC, 2001.

[19] K. S. Thyagarajan, Still Image and Video Compression with MATLAB, Wiley, 2011.

[20] P. Reu, Stereo-Rig Design: Lighting-Part 5, Society for Experimental Mechanics, 37, 2013, 12.

[21] https://www.ix-cameras.com/high-speed_camera_ix_cameras.php 